\title{
Aspectos sociales de la muerte materna: análisis de 5 años en el Hospital General de Occidente. Jalisco, México
}

\author{
Martha Leticia Mejía M. ${ }^{1}$, Roberto Carlos Ortiz V. ${ }^{2}$, Jorge Laureano ${ }^{3}{ }^{3}$, Elizabeth Clotilde \\ Guillermina Alcántara $\mathrm{H}^{4}$, María del Carmen López $\mathrm{Z}^{5}$, Elisa Gil $\mathrm{H}^{6}$ \\ ${ }^{1}$ Profesor del Departamento de Salud Pública, Universidad de Guadalajara, Secretaría de Salud. ${ }^{2}$ Coordinador del área \\ de obstetricia, Hospital General de Occidente. ${ }^{3}$ Asesor de investigación cualitativa, Departamento de Investigación Es- \\ tatal, Secretaría de Salud. "Profesor investigador titular "c", coordinador de patrimonio de la 3ra. División, Universidad \\ de Guadalajara. ${ }^{5}$ Profesor investigador titular "c", Universidad de Guadalajara. ${ }^{6}$ Asesor de investigación cuantitativa, \\ Departamento de Investigación Estatal, Secretaría de Salud.
}

\section{RESUMEN}

Objetivo: Analizar los aspectos sociales de las muertes maternas ocurridas en el Hospital General de Occidente, Jalisco, México (2006-2010). Método: Se recurrió al archivo del hospital para identificar los expedientes de las muertes maternas ocurridas durante el periodo de estudio. Las fuentes de información fueron certificados de defunción, autopsia verbal, cuestionario confidencial y dictamen de las muertes. Se utilizó estadística descriptiva y medidas de tendencia central para el análisis de la información. Resultados: Se identificaron 22 muertes maternas, evidenciando un aumento de las muertes maternas durante el periodo de estudio. La edad media fue de 28,27 años, el $68,27 \%$ se dedicaba actividades del hogar, $45 \%$ vivían en unión libre, $55 \%$ no tenían registro de tener seguridad social, el $50 \%$ poseían estudios de primaria o menos, y solo $77,2 \%$ presentaba control prenatal. Conclusiones: La presencia de factores sociales, que además de los clínicos, y que inciden en la muerte materna, deben ser conocidos por el personal del servicio de obstetricia, y deben ser considerados para el diseño e implementación de acciones para disminuir la muerte materna en todos los niveles de atención.

\section{PALABRAS CLAVE: Muerte materna, cultura, servicios de salud, factores sociales, inequidad en salud}

\section{SUMMARY}

Objective: Analyze the social aspects of the maternal mortality occurred in the Hospital General de Occident, Jalisco, Mexico (2006-2010). Method: It is resorted the archive of the hospital to identify files of maternal mortality during the study period. The sources of information were death certificates, verbal autopsy, confidential questionnaire and opinion of the deaths. We used descriptive statistics and measures of central tendency for the analysis of the information. Results: We identified 22 maternal deaths, demonstrating an ascending trend of maternal mortality during the study period. The mean age was 28.27 years old, $68.27 \%$ was dedicated to the home, $45 \%$ lived in free union, $55 \%$ had no record of social security, $50 \%$ had a primary education or less, and only $77.2 \%$ had prenatal control. Conclusions: The presence of social factors, in addition to clinicians, and that influence maternal death should be known for the obstetrics service personnel, and should be considered for the design and implementation of actions to reduce maternal death at all levels of care.

KEY WORDS: Maternal mortality, culture, health services, social factors, health inequities 


\section{INTRODUCCIÓN}

La muerte materna, es una expresión de injusticia social. El $99 \%$ de estas mujeres viven y mueren en países en desarrollo [1,2], lo que traduce que las mujeres pobres presentan un mayor riesgo de morir en el embarazo, parto o puerperio [3], por lo que su análisis, implica considerar además de los factores biológicos y clínicos, aquellos aspectos culturales, económicos y geográficos que determinan que mujeres embarazadas de una misma sociedad mueran de manera inequitativa, buscando con ello que los servicios de obstetricia, brinden una atención a partir del análisis sobre la influencia que tienen los aspectos sociales en la muerte materna, ejercicio que busca una atención equitativa y de calidad [4].

México, para el 2010 presentó una razón de mortalidad materna de 48,9/100.000 nacidos vivos, y Jalisco de $37,9 / 100.000$ nacidos vivos, lo anterior pese a las acciones desarrolladas a través de los programas de salud, mencionando que estas no siguen trayectorias lineales, debiéndose también al comportamiento de los aspectos sociales, los cuales retrasan la disminución esperada, teniendo que dirigir los esfuerzos a las unidades que brindan atención a poblaciones marginadas $[1,5,6]$.

El Hospital General de Occidente, es la unidad de referencia de tercer nivel en los Servicios de Salud Jalisco, por lo que analizar los aspectos sociales de las muertes maternas, permite tener otros elementos para tomar acciones concretas de las autoridades correspondientes.

El objetivo de este estudio es analizar los aspectos sociales de la muerte materna durante un período de 5 años en el hospital General de Occidente, Jalisco, México.

\section{MATERIAL Y MÉTODOS}

Estudio descriptivo, retrospectivo y transversal, donde se analizaron los datos relacionados a los aspectos sociales de todas las muertes maternas ocurridas durante el período 2006 al 2010 en el Hospital General de Occidente de los Servicios de Salud Jalisco. La información se obtuvo del archivo del hospital para utilizar como fuente de información los certificados de defunción, la autopsia verbal, el cuestionario confidencial y el dictamen de las muertes maternas. Los datos fueron procesados a través del programa estadístico Epi Info versión 16.0, utilizando estadística descriptiva y medidas de tendencia central para el análisis de la información.

\section{RESULTADOS}

Durante el periodo de estudio, hubo 22 muertes maternas, observándose una tendencia ascendente (Tabla I).
El promedio de edad de las fallecidas, fue de 28,27 años (rango: 16-38 años), la distribución de las edades por rango de edad materna al momento del fallecimiento se presenta en la Tabla II.

En la Tabla III se describen los aspectos sociales presentes en las muertes maternas, elementos que permiten hacer un análisis de aquellos factores que aun cuando no es posible incidir de manera directa por parte de la atención brindada por el personal de salud del servicio de obstetricia, son determinantes para que se presente la muerte materna.

El $26,3 \%(n=5)$ tenían como antecedente la utilización de algún método de planificación familiar, no utilizaban el $31,8 \%(n=7)$ y $45,5 \%(n=10)$ se ignora la información. El $77,2 \%(n=17)$ de las muertes registradas tenían datos de tener al menos un control prenatal por médico general o especialista, sin control prenatal el $9 \%(n=2)$ y se ignora en el $13,6 \%(n=3)$.

De las muertes notificadas como directas $(n=13)$, la principal causa descrita fue la hemorragia obstétrica con el $46,1 \%$, mientras que en las muertes indirectas los problemas pulmonares fueron la principal causa $(n=9)$ con el $33,4 \%$ de los fallecimientos (Tabla IV).

Tabla I

TOTAL DE MUERTES MATERNAS, 2006-2010

\begin{tabular}{ccc}
\hline Año & $\begin{array}{c}\text { Muertes maternas } \\
(\mathrm{n})\end{array}$ & $\begin{array}{c}\text { Frecuencia } \\
(\%)\end{array}$ \\
\hline 2006 & 3 & 13,6 \\
2007 & 4 & 18,1 \\
2008 & 3 & 13,6 \\
2009 & 5 & 22,7 \\
2010 & 7 & 31,8 \\
\hline
\end{tabular}

Fuente: Certificado de defunción y autopsia verbal de las muertes maternas, Hospital General de Occidente. Jalisco, México.

\section{Tabla II \\ EDAD AL MOMENTO DE LA MUERTE MATERNA}

\begin{tabular}{ccc}
\hline $\begin{array}{c}\text { Edad materna } \\
\text { (años) }\end{array}$ & $\begin{array}{c}\text { Muertes maternas } \\
(\mathrm{n})\end{array}$ & $\begin{array}{c}\text { Frecuencia } \\
(\%)\end{array}$ \\
\hline$<20$ & 2 & 9,1 \\
$20-34$ & 16 & 72,7 \\
$\geq 35$ & 4 & 18,2 \\
\hline
\end{tabular}

Fuente: Certificado de defunción y autopsia verbal de las muertes maternas, Hospital General de Occidente. Jalisco, México. 
Tabla III

ASPECTOS SOCIALES DE LAS MUERTES MATERNAS. HOSPITAL GENERAL DE OCCIDENTE, JALISCO MÉXICO. 2006-2010

\begin{tabular}{ccc}
\hline Variable & $\begin{array}{c}\text { Muertes } \\
\text { maternas } \\
(\mathrm{n})\end{array}$ & $\begin{array}{c}\text { Frecuencia } \\
(\%)\end{array}$ \\
\hline
\end{tabular}

\section{Ocupación}

Hogar

Empleada

Comerciante

Estudiante

\section{Estado civil}

Soltera

Casada

Unión libre

Separada

$\begin{array}{cc}15 & 68,1 \\ 4 & 18,1 \\ 2 & 9,0 \\ 1 & 4,0\end{array}$

\section{Derechohabiencia}

$\mathrm{Si}$

No

\section{Escolaridad}

Analfabeta

Primaria incompleta

Primaria completa

Secundaria

Preparatoria o equivalente
10

12

$\begin{array}{cc}3 & 14,0 \\ 8 & 36,0 \\ 10 & 45,0 \\ 1 & 5,0\end{array}$

5,0

4,6

9,0

36,4

45,4

4,6
Fuente: Autopsia verbal, cuestionario confidencial y dictamen de las muertes maternas, Hospital General de Occidente. Jalisco, México.

\section{DISCUSIÓN}

Los resultados de este estudio hacen evidente un comportamiento ascendente en las muertes maternas. El análisis de los aspectos sociales de estas, permitirá en primera instancia, la reflexión sobre la creación y operación de los programas y las estrategias utilizadas, los actores que participan y la manera en que se comunican, identificando oportunidades de mejora y la generación de nuevas modalidades en la organización y aplicación, específicamente dentro de los servicios de atención obstétrica hospitalaria, justificado en la importancia que este problema de salud pública tiene, posicionándolo como un indicador fundamental del desarrollo social de un país $[7,8]$.

Debemos resaltar el promedio de edad de las muertes maternas que correspondió a 28,27 años, que corresponde a mujeres económicamente activas y jóvenes, concordante con los resultados de
Tabla IV

\section{CAUSAS DE MUERTES MATERNAS. HOSPITAL GENERAL DE OCCIDENTE, JALISCO MÉXICO. 2006-2010}

\begin{tabular}{ccc}
\hline Causas & $\begin{array}{c}\text { Muertes } \\
\text { maternas } \\
(\mathrm{n})\end{array}$ & $\begin{array}{c}\text { Frecuencia } \\
(\%)\end{array}$ \\
\hline
\end{tabular}

\section{Directas}

Hemorragia obstétrica

$6 \quad 46,1$

Toxemia del embarazo +

5

38,5

hemorragia

Aborto + hemorragia

Septicemia

1

7,7

1

7,7

Indirectas

Problemas Pulmonares $\quad 3 \quad 33,4$

Cardiopatía

22,2

Alteraciones Metabólicas

Alteraciones Renales

Lupus

Alteraciones Vasculares

11,1

11,1

11,1

11,1

Fuente: Autopsia verbal, cuestionario confidencial y dictamen de las muertes maternas, Hospital General de Occidente. Jalisco, México.

una investigación realizada en México en el Hospital General Regional 36 de Puebla con un promedio de 28,8 años [9], de manera contraria, se encuentran diferencias con otras investigaciones en cuanto al promedio de edad, una de ellas realizadas en Cuba en el Hospital docente Ginecobstétrico "Mariana Grajales", estudio realizado del 2001 al 2010 donde la edad más representada fue de 36 años [10], y otra realizada en Perú en la Región La Libertad, donde la muerte ocurrió en el $12 \%$ en adolescentes y el $29 \%$ en mujeres mayores de 35 años [11].

La trascendencia de la edad de muerte materna para este estudio, radica en que no son los extremos de la edad fértil, considerados un factor de riesgo de muerte materna [12], por lo que es necesario a través de una investigación específica, hacer otros acercamientos que permitan hacer evidente las razones o causas de este comportamiento.

Debemos destacar que la ocupación de las fallecidas en el $68,1 \%$ se dedicaron al hogar, no encontrando a ninguna mujer profesionista ya que el $95,4 \%$ de estas mujeres tenían estudios de secundaria o menos. Esto hace evidente lo que la Organización Mundial de la Salud ha señalado en su informe titulado "La educación y la salud están íntimamente unidas", asumiendo que las mujeres que tienen un mejor nivel de estudios, puede acceder a 
una mejor situación laboral y familiar, con buenas prácticas de atención en salud, escenario contrario para aquellas mujeres con un nivel de estudio más bajo y expectativa laboral reducida [13].

Al respecto, otras investigaciones han descrito que el bajo nivel educativo se asocia a un mayor riesgo de muerte durante el periodo de gestación, señalando que una mujer sin preparación no reconoce los signos de alarma o busca tardíamente la atención hospitalaria, haciendo evidente la necesidad de focalizar las acciones de educación para la salud desde el primer nivel de atención donde las pacientes llevan su control prenatal [11].

El $55 \%$ de las madres fallecidas no contaban con algún tipo de derechohabiencia, por lo que la posible falta de cobertura del sistema actual de salud en México, puede ser un problema aun presente, necesitando cruzar los datos con otras fuentes de información. Este aspecto es importante si se considera el bajo nivel de estudios y no tener ningún tipo de seguridad social, incrementa los riesgos de una muerte materna debido a las dificultades de acceso oportuno a las unidades de atención, la capacidad de decisión de la mujer con respecto a su salud. Se recomienda llevar a cabo una investigación representativa a la población de la entidad, que permita evidenciar el impacto que ha tenido el Seguro Popular y la muerte materna en las comunidades con estas características, contrastado con las muertes ocurridas en hospitales que atienden a población con mejor nivel económico [14].

Junto a lo anterior, el contar con seguridad social, no se puede traducir en que la mujer no terminará en una estadística mas de muerte materna, ya que las pacientes de este estudio con seguridad social registrada, formaron parte de las cifras, haciendo evidente que detrás de la salud de la mujer hay un contexto social e histórico que influye de manera directa o indirecta en la ocurrencia de estos eventos no deseados en salud [15].

Que solo el $26,3 \%$ tengan registro de uso de algún método de planificación familiar y un 31,5\% no usar ningún tipo de método, debe de ser un punto de consideración por las autoridades, resaltando que la planificación familiar es una estrategia importante en la lucha contra la mortalidad materna, ejemplo de ello es que para Chile, el control de la fecundidad es una de las intervenciones más importantes para controlar este problema de salud pública [16].

Uno de los aspectos que más se ha descrito en diferentes estudios, ha sido que las mujeres llevaron atención prenatal; para este estudio en el $77,2 \%$ de las muertes, hubo registro de haberlo llevado, al compararlo a cifras con otros autores, se encontró un porcentaje menor a los resultados de ellos; ejemplo es un estudio realizado en Chile, reportando un acceso a control prenatal en $90 \%$ de las muertes maternas, además en este estudio se observa que el $74 \%$ de los casos conto con atención por médico especialista [16].

Con estos datos debería de analizarse la calidad con que se brinda la atención, así como el número de consultas médicas, lo anterior señalando que la atención prenatal se considera que reduce la morbi-mortalidad materna, permitiendo además identificar factores de riesgo, posibilitando establecer acciones preventivas y oportunas durante el embarazo [17]. Dentro de la toma de decisiones sobre el llevar o no un control prenatal, también se ha señalado que en los grupos de más bajos recursos y con niveles de estudios básicos o nulos, las creencias y actitudes durante su embarazo, responde en gran medida a patrones que culturalmente son determinantes en el comportamiento de una población.

Algo que evidencia esta situación, es haber encontrado mujeres adolescentes y con alguna patología agregada, representando un factor de riesgo para considerar una posible muerte materna, situación que hace necesario hacer una análisis de las intervenciones realizadas en primer nivel de atención, desde la vigilancia durante su embarazo, búsqueda de atención oportuna en una unidad hospitalaria, pero sobre todo, lo referente a las acciones de educación y promoción de la salud, proponiendo el acompañamiento del personal de salud o personas capacitadas en la toma de conciencia de estas mujeres para decidir con conocimiento sobre las complicaciones para ella y su bebe, por no ser atendida oportunamente, implicando el riesgo de una muerte materna [18].

También el que de los 22 casos de muerte materna, el 33,3\% fueron indirectas, es una situación de consideración, ya que estos son aspectos prevenibles para la paciente, relacionado a la toma de decisiones sobre su auto-cuidado, por las implicaciones que le trae el embarazarse en ciertas condiciones no favorables para su salud, señalando nuevamente al nivel de estudios y económico como aspectos determinantes para que se presenten estos casos.

Debemos tomar en cuenta que la conducta de las personas está determinada por su contexto cultural, esto puede llevarlas a tener prevención o no, respecto a su condición reproductiva, siendo en su conjunto los aspectos sociales los determinantes de la muerte materna, influyendo en la conducta de la mujer embarazada. Esto es señalado por algunos autores que el contexto cultural es clave para entender temas como las creencias, respecto al cuidado previo al embarazo y durante su control prenatal [13].

Al comparar los datos de causas directas e indirectas, se encuentran datos similares con investigaciones realizadas en el Hospital General de Matamoros "Dr. Alfredo Pumarejo Lafaurie", durante un periodo de 10 años publicada en el 2010 y otra 
llevada a cabo en el Hospital de Gineco-Obstetricia del Centro Médico Nacional de Occidente, durante una revisión de 12 años, publicada en 1999.

En un estudio realizado en el Estado de México del 2004 al 2006, se encontró una diferencia en porcentaje entre las muertes maternas directas e indirectas más amplio que en nuestros resultados, situación similar también en otra investigación realizada en Veracruz en la Delegación Norte del IMSS en el 2009 , donde encontraron un $32 \%$ de muertes directas y $68 \%$ por causa indirecta [19].

Los aspectos sociales de las muertes maternas de este hospital al igual que de aquellos con características similares, deben de asumirse como aquellos elementos de la estructura social que condicionan la posición de la mujer dentro de la sociedad, teniendo un efecto directo en la distribución de los problemas durante el embarazo o de los factores protectores durante el mismo, sobre todo cuando gran parte de estas muertes están directamente relacionadas con la accesibilidad, la oportunidad de las intervenciones, los costos y la calidad de los servicios obstétricos y perinatales [15], aspectos que al ser analizados de manera integral, permitan la redirección y creación de los programas y estrategias que impacten en la problemática.

Por lo anterior, es importante que los procesos de mejora continua de las unidades hospitalarias y de primer nivel de atención, se contextualicen con un panorama más amplio de los elementos que comúnmente se debaten en la temática de muerte materna, incorporando las causas de las causas en cada una de las muertes maternas presentadas, pues si no se llega a comprender el papel que juegan los aspectos sociales, cualquier solución que se proponga para prevenir la mortalidad materna, podría ser superficial [20].

Se recomienda también involucrar otros sectores en las mesas de discusión y toma de decisiones, tales como el educativo, desarrollo social, económico y fomento cultural, lo anterior con la posibilidad de incidir en este problema de salud pública no solo con las intervenciones del Sector Salud, sino que también con la búsqueda de mejorar las condiciones de vida de la población, trabajo, acceso a educación e información, para que en conjunto a mediano y largo plazo, se incida desde sus inequidades a nivel local de cada comunidad con enfoques en diferentes niveles de jerarquía, no solo dejando como responsable al sector salud para disminuir esta problemática [15].

Para poder tener un marco de análisis más amplio sobre los aspectos sociales de la muerte materna y ser estudiados con mayor profundidad, se recomienda retomar otras estrategias metodológicas, sobre todo de tipo cualitativo que desgeneraliza las causantes de la mortalidad materna por la diversidad de los hechos sociales y culturales que influyen en cada caso, contemplando el perfil de la mujer, ubicación socio-demográfica y económica, rol social, significación del embarazo, apoyo o exigencias recibida de sus familiares y redes sociales, relaciones inequitativas de género, violencia, atención tradicional del embarazo, factores psicológicos, entre otros [21].

\section{CONCLUSIONES}

Podemos concluir que para los servicios de obstetricia, al hacer este análisis, no se intenta restar la importancia a las recomendaciones médicas sobre el control prenatal, si no que se busca hacer evidente y reconocer los causantes sociales al igual que culturales detrás de cada muerte materna, algo que complemente el total de información para incidir en este problema multicausal, intervenciones que señalan que la situación última de una mujer embarazada, el término del embarazo al igual que el posparto, no solo dependerá de la calidad clínica con que brinde la atención [22].

Las muertes maternas de este hospital, son claro ejemplo del grado de limitaciones del sector salud en general y de manera particular, el servicio de obstetricia, para responder en forma efectiva a un problema que sobrepasa la capacidad de respuesta institucional, teniendo que empezar a reconocer y tomar en cuenta las inequidades en salud y sus determinantes dentro de la población que se atiende, ya que esta estructura enferma y mata de manera desigual a una misma población y de una misma causa de deceso: muerte materna [23].

\section{REFERENCIAS}

1. Lozano AR, Núñez URM, Duarte GMB, Torres PLM. Evolución y tendencias de largo plazo de la mortalidad materna en México: análisis de factibilidad y de efecto potencial de intervenciones seleccionadas para el cumplimiento de las metas del milenio. En: Zuñiga HE. México ante los desafíos de desarrollo del milenio. México: Editorial Consejo Nacional de Población, 2005; 167-92.

2. Mortalidad materna. Nota descriptiva No. 348. Organización Mundial de la Salud. Mayo 2012. Disponible en:http://www.who.int/mediacentre/factsheets/fs348/ es/\#. Acceso el 15 de Agosto de 2013.

3. Briones GC, Díaz de León PM, Gutiérrez VMC, Rodríguez RM. Los paradigmas en la muerte materna. Rev Asoc Mex Med Crit y Ter Int 2010;24(4):185-9.

4. Borrell C, Malmusi D. La Investigación sobre los determinantes sociales y las desigualdades en salud: Evidencias para la salud en todas las políticas. Informe SESPAS 2010.Gaceta Sanitaria 2010;24(1):10-6.

5. Cubo de Muertes Maternas 2002 a 2011. Dirección General de Información en Salud. México: Gobierno Federal, 2012. Disponible en: http://pda.salud.gob.mx/ cubos/cmaternas.html. Acceso el 20 de Julio de 2013.

6. Gobierno Federal de México. Programa Nacional de Salud 2007 - 2012. Por un México sano: construyendo alianzas para una mejor salud. Secretaria de Salud: México, 2007. 
7. Gobierno Federal de México. Manual de atención del embarazo, parto y puerperios seguros, recién nacido sano. Secretaria de Salud: México, 2001.

8. Grupo de trabajo regional interagencial para la reducción de la mortalidad materna. Reducción de la mortalidad y la morbilidad materna: Consenso Estratégico Interagencial para América Latina y el Caribe. Washington D.C,: PAHO, 2003. Disponible en: http:// publicaciones.ops.org.ar/publicaciones/piezas $\% 20$ comunicacionales/cdcofesa/pdf/redMMymorbilidad_ spanish.pdf. Acceso el 20 de Julio de 2013.

9. Hernández PJA, Ramos AG, López CA. Análisis de casos de muerte materna en un periodo de 10 años. Ginecol Obstet México 2007;75:61-7.

10. Suárez GJA, Gutiérrez MM, Lorenzo GBC. Problemática de la muerte materna en Villa Clara. Rev Cubana Cir 2011;50(4):28-35

11. Santos L, Luna Victoria F, González M. Mortalidad materna: factores determinantes modificables mediante políticas públicas en la región La Libertad. UCV - Scientia 2010;2(1):22-33.

12. Donoso E, Villarroel L. Edad materna avanzada y riesgo reproductivo. Rev Med Chile 2013;131(1):55-9.

13. La educación y la salud están íntimamente unidas. Margaret Ch. Organización Mundial de la salud. Nueva York. 2010. Disponible en: http://www.who.int/dg/ speeches/2010/educationandhelath_20100920/es\# Acceso el 2 Agosto de 2013.

14. Karam MA, Bustamante $P$, Campuzano M, Camarena A. Aspectos sociales de la mortalidad materna. Estudio de caso en el Estado de México. Medicina Social 2007;2(4):205-11.
15. Eslava SJ. Inequidades sociales en la salud materna. Rev Colombiana Obstet Ginecol 2010;61(3):193-6.

16. Donoso E. Muerte materna en Chile por eclampsia. Rev Chil Obstet Ginecol 2010;75(5):281-3.

17. Sánchez NHR, Pérez TG, Pérez RP, Vázquez NF. Impacto del control prenatal en la morbilidad y mortalidad neonatal. Rev Med IMMSS 2005;43(5):377-380.

18. Donoso E. Tras el cumplimiento del 5to objetivo del milenio: mortalidad materna, Chile 2007. Rev Chil Obstet Ginecol 2010;75(1):1-2.

19. Caballero LLA, Muñoz RMR, Soler HE, Blanco CAV, Aguilar BMR. Mortalidad Materna. Experiencia de cinco años en la Delegación Veracruz Norte del IMSS. Ginecol Obstet Mex 2009;77(11):508-11.

20. Gobierno Federal de México. Programa de Acción Específico Sistema Integral de Calidad en Salud SICALIDAD, 2007-2012. Secretaria de Salud: México; Disponible en: http://www.calidad.salud.gob.mx/doctos/ calidad/pa_sicalidad.pdf. Acceso el 15 Mayo de 2013.

21. Romero PI, Sánchez MG, Romero LE, Chávez CM. Muerte materna: una revisión de la literatura sobre factores socioculturales. Perinatol Reprod Hum 2010;24(1):45-50.

22. Chávez CM, Sánchez MG, Romero LE, Torres CJL, González PI. Propuesta metodológica para el estudio de muerte materna desde la perspectiva antropológica y social. Perinatol Reprod Hum 2010;24(1):67-75.

23. Álvarez CLS. Los determinantes sociales de la salud: más allá de los factores de riesgo. Gerencia y Políticas de Salud Bogotá. Rev Gerenc Polit Salud, Bogotá (Colombia) 2009;8(17):69-79. 